\title{
A Generalized Convergence Result for the Graph-Based Ant System Metaheuristic
}

\author{
Walter J. Gutjahr \\ Department of Statistics, Operations Research and Computer Science \\ University of Vienna
}

\begin{abstract}
It is shown that on fairly weak conditions, the current solutions of a metaheuristic following the ant colony optimization paradigm, the Graph-Based Ant System, converge with a probability that can be made arbitrarily close to unity to one element of the set of optimal solutions. The result generalizes a previous result by removing the very restrictive condition that both the optimal solution and its encoding are unique (this generalization makes the proof distinctly more difficult), and by allowing a wide class of implementation variants in the first phase of the algorithm. In this way, the range of application of the convergence result is considerably extended.
\end{abstract}

\section{Introduction}

Modern information-technological systems get more and more complex, which creates an urgent demand for efficient heuristics for solving search and optimization problems. Heuristics tailored to the solution of particular problems are required, but there is also a growing interest into socalled metaheuristics that promise to be applicable, after suitable specifications, to large classes of different problem types. It is not surprising that some of these metaheuristics are inspired by nature, since natural evolution processes can be considered, from a certain point of view, as mechanisms constantly adapting and improving the functionality of very complex (biological) systems.

A well-established paradigm within the class of nature-inspired metaheuristics is that of genetic algorithms ([16], [18]). Recently, also another paradigm within this class has found considerable attention: the ant colony optimization approach. Introduced in 1991 by Dorigo, Maniezzo and Colorni ([7], [4]), this approach has developed in the meantime into a powerful, general and efficient metaheuristic with applications in various fields (see, e.g., [6], [5], [17]). In particular, the whole range of solving combinatorial optimization problems heuristically can be covered by this metaheuristic. A feature making it especially attractive is that it is wellsuited for implementations on parallel or distributed systems [3], performing well already on single-processor systems.

Whenever a heuristic algorithm for an optimization problem allows the investment of an arbitrary amount of computation time, improving the current solution as more computation time is spent, one wishes to know what is likely to happen in the long run. Will the current solution get closer and closer to an optimal solution, or is it possible that there will always remain a gap to the optimum? For a prominent metaheuristic inspired by physical processes, 
simulated annealing (SA), it has been shown that the probability distribution of the current solution converges to the uniform distribution on the set of optimal solutions (see, e.g., [12], [15], [1]). Similar results would be desirable for other metaheuristics.

In [13], a first step towards a convergence result for the ant colony optimization approach has been achieved: For a special metaheuristic algorithm of this type, Graph-Based Ant System, covering the range of static combinatorial optimization problems, it has been shown that on four particular conditions (which will be outlined here at the beginning of section 3), the current solutions converge with a probability that can be made arbitrarily close to one to the optimal solution. This convergence result is weaker than that for SA (where convergence holds with probability one), but the more serious drawback lies in one of the conditions which requires not only that there is a unique optimal solution, but even that this solution is encoded within the framework in a unique way. In many applications, this cannot be guaranteed. So it seems highly desirable to remove or at least to relax the uniqueness condition.

As it turns out, however, this is not easy at all: We shall show in the present article that the uniqueness condition can be totally dropped, but at the price of making the convergence proof considerably more involved. Moreover, we shall relax another of the four conditions of the result in [13], requiring a specific parameter update strategy. We shall show that the result remains valid if this strategy is applied at least in the final phase of the algorithm, after a phase where the update mechanism can be chosen arbitrarily within the given framework, including, e.g., the rank-based variant suggested in [2]. Contrary to the removal of the uniqueness condition, this second generalization causes no serious difficulties.

The rest of the article is organized as follows: In section 2, we present our formal framework, the Graph-Based Ant System, and illustrate the relevance of the uniqueness condition by a concrete example. Section 3 contains the mathematical result and its proof, which is performed with the help of seven lemmas. Section 4, finally, contains some short concluding remarks.

\section{The Algorithm}

The description below of our general algorithm, the Graph-based Ant System (GBAS), follows closely the presentation in [13]. The reader should be aware, however, of some extensions in the definitions.

GBAS is based on the representation of a feasible solution of a combinatorial optimization problem as a walk in a directed graph, which we call the "construction graph":

Definition 2.1. Let an instance of a combinatorial optimization problem be given. By a construction graph for this instance, we understand a directed graph $\mathcal{C}=(\mathcal{V}, \mathcal{A})$ together with a function $\Phi$ with the following properties:

(1) In $\mathcal{C}$, a unique node is marked as the so-called start node.

(2) Let $\mathcal{W}$ be the set of (directed) walks $w$ in $\mathcal{C}$ satisfying the following conditions:

(i) $w$ starts at the start node of $\mathcal{C}$.

(ii) $w$ contains each node of $\mathcal{C}$ at most once.

(iii) The last node on $w$ has no successor node in $\mathcal{C}$ that is not already contained in $w$ (that is, $w$ cannot be extended without violating (ii)). 
Then $\Phi$ maps the set $\mathcal{W}$ onto a set $\mathcal{S}$ of solutions of the given problem instance containing all feasible solutions. In other words: To each walk $w$ satisfying (i) - (iii), there corresponds (via $\Phi$ ) a solution in $\mathcal{S}$, and to each solution in $\mathcal{S}$ (in particular: to each feasible solution), there corresponds (via $\Phi^{-1}$ ) at least one walk satisfying (i) - (iii).

As it can be seen from this definition, a construction graph $(\mathcal{C}, \Phi)$ specifies a particular encoding of the solutions as "walks". The objective function value of the walk is set equal to the objective function value of the corresponding solution of the original problem, if this solution is feasible, and to infinity otherwise. We assume throughout this paper that the optimization problem under consideration is formulated as a minimization problem, that is, the objective function represents costs. Usually, there are several ways to design a construction graph for a given combinatorial optimization problem; a standard way is outlined in [13].

Let us now describe GBAS as an extension of Ant System [7], [4], [8]. GBAS contains the following components:

1. A construction graph $(\mathcal{C}, \Phi)$ according to Definition 2.1 .

2. A set $A_{1}, \ldots, A_{S}$ of agents $^{1}$. Each agent performs a random walk with certain transition probabilities (see component 3 below) in the construction graph. In a multiprocessor system, the walk of each agent may be computed on a separate processor (cf. [3]). In a single-processor system, the moves of $A_{1}, \ldots, A_{S}$ are computed sequentially. A time period in which each agent performs a walk (consisting of several single moves) through the construction graph will be called a cycle. An application of the Ant System consists of several cycles $1, \ldots, M$; the number $M$ of cycles may be fixed in advance or be determined at a later time during the execution of the algorithm.

3. Transition probabilities for the random moves of the agents during each cycle. Let $u=\left(u_{0}, \ldots, u_{t-1}\right)$ denote the partial walk an agent has already traversed before its $t$ th transition step in a fixed cycle $n$, where $u_{0}, \ldots, u_{t-1}$ are node indices in the construction graph $\left(u_{0}\right.$ referring to the start node). We write $l \in u$ if node $l$ is contained in the partial walk $u$, and $l \notin u$ otherwise. Moreover, let $\mathcal{A}$ be the set of arcs in the construction graph. Then the general form of the transition probabilities is

$$
p_{k l}(n, u)=\frac{\left[\tau_{k l}(n)\right]^{\alpha} \cdot\left[\eta_{k l}(u)\right]^{\beta}}{\sum_{r \notin u,(k, r) \in \mathcal{A}}\left[\tau_{k r}(n)\right]^{\alpha} \cdot\left[\eta_{k r}(u)\right]^{\beta}},
$$

if $l \notin u$ and $(k, l) \in \mathcal{A}$, and

$$
p_{k l}(n, u)=0
$$

otherwise. Therein, $p_{k l}(n, u)$ denotes the probability that a fixed agent having already traversed a partial walk $u=\left(u_{0}, \ldots, u_{t-2}, u_{t-1}=k\right)$ in the current cycle $n$, moves from node $k$ (its current position) to node $l$. (Note that this probability is only defined if $k=u_{t-1}$.) The numbers $\tau_{k l}(n)$ are called "trail levels" (see component 4 below), and the

\footnotetext{
${ }^{1}$ Despite the important role the ant metaphor has played for the development of the class of heuristics to which our approach belongs, we prefer to call our computational units "agents" rather than "ants": The ant metaphor suggests a relatively simple behavior, whereas our agents may as well act in a very "intelligent" way, using, e.g., heuristics based on sophisticated methods of mathematical programming.
} 
numbers $\eta_{k l}(u)$ are called "attractiveness values" (see component 5 below) ${ }^{2} . \alpha \geq 0$ and $\beta \geq 0$ are parameters.

At the beginning of each cycle, each agent is positioned in the start node of the construction graph. In the $t$ th transition step of the current cycle, each agent $A_{s}$ performs a single random move in the construction graph, controlled by the transition probabilities $p_{k l}(n, u)$, where $u$ is the partial walk $A_{s}$ has already traversed (that is, each agent has a particular $u$ ). If, for a fixed agent $A_{s}, p_{k l}(n, u)=0$ holds for all nodes $l$ before the $t$ th transition step, then agent $A_{s}$ has completed his walk in the current cycle $n$. According to Definition 2.1, this walk determines a solution of the given optimization problem.

4. An array of trail levels $\tau_{k l}$, where $\tau_{k l} \geq 0$ is assigned to arc $(k, l)$ in the construction graph. The trail levels usually change from cycle to cycle (see below), so their dependence on the cycle index $n$ can be represented in the form $\tau_{k l}(n)$. At the beginning of cycle 1 , we set $\tau_{k l}=1 /|\mathcal{A}|(|\mathcal{A}|$ being the number of arcs) for each arc $(k, l)$. At the end of each cycle $n(n=1,2, \ldots, M-1)$, the following update rule is applied. First, for each agent $A_{s}$ and each arc $(k, l)$, a value $\Delta \tau_{k l}^{(s)}$ is determined as a function of the solutions assigned to the walks of the agents in the current cycle $n$ : Suppose these solutions have cost values (objective function values) $f_{1}, \ldots, f_{S}$. We set for each $\operatorname{arc}(k, l)$ and each $s \in\{1, \ldots, S\}$ :

$$
\Delta \tau_{k l}^{(s)}= \begin{cases}\varphi_{s}\left(f_{1}, \ldots, f_{S}\right), & \text { if agent } A_{s} \text { has traversed arc }(k, l), \\ 0, & \text { otherwise. }\end{cases}
$$

Therein, each $\varphi_{s}$ is a nonnegative reward function ${ }^{3}$ which is nonincreasing in the corresponding variable $f_{s}$ and may depend on the walks of the agents in the cycles $1, \ldots, n-1$. Let

$$
C=\sum_{(k, l) \in \mathcal{A}} \sum_{s=1}^{S} \Delta \tau_{k l}^{(s)}
$$

Now, if $C=0$, we set

$$
\tau_{k l}(n+1)=\tau_{k l}(n)
$$

for all arcs $(k, l)$, that is, the values $\tau_{k l}$ are the same in cycle $n+1$ as in cycle $n$. If, on the other hand, $C>0$, we set

$$
\tau_{k l}(n+1)=(1-\rho) \tau_{k l}(n)+\rho \Delta \tau_{k l},
$$

where

$$
\Delta \tau_{k l}=\frac{1}{C} \sum_{s=1}^{S} \Delta \tau_{k l}^{(s)}
$$

As in [8], the number $\rho$ will be called the evaporation factor. It is easily verified from (5), (6) and (4) that the sum of trail levels, $\sum_{(k, l) \in \mathcal{A}} \tau_{k l}(n)$, always remains equal to one. Forcing the sum of the values $\tau_{k l}(n)$ to be one is a re-normalization which favors the

\footnotetext{
${ }^{2}$ In [13], the terms "pheromone values" and "desirability values" have been used for the numbers $\tau_{k l}(n)$ and $\eta_{k l}(u)$, respectively. We use here the terminology of [17] which is not that closely related to the ant metaphor (cf. Footnote 1).

${ }^{3}$ Note that this formalism covers the rank-based trail level update, as introduced in [2], as well as the classical proportional update.
} 
numerical stability of the algorithm by preserving the overall order of magnitude of the trail level values.

The trail level update rule above may be interpreted as follows: If no walk is rewarded, everything remains constant. Otherwise, due to "evaporation", only an amount of $1-\rho$ of the sum of the trail levels, remains on the graph after cycle $n$. The remaining amount of $\rho$ is the "budget" for rewarding the walks traversed in cycle $n$ according to their respective objective function values. Each agent $A_{s}$ can be imagined to report by which "bonus" $\Delta \tau_{k l}^{(s)}$ he wants to reward his walk in cycle $n$. The actual trail level increments result by distributing the total budget of $\rho$ proportionally to the reported values $\Delta \tau_{k l}^{(s)}$.

By the described update rule, the trail levels of "successful" arcs of the construction graph are increased, such that they are traversed more often by the agents in the future. By setting $\rho=0$, the influence of the cost function on the walks of the agents is switched off. If, in addition, the influence of the attractiveness values $\eta_{k l}(u)$ is switched off by setting $\beta=0$, then we obtain random search by a ("blind") random walk in the construction graph, which, in this way, turns out to be a special case of GBAS.

5. An array of attractiveness values $\eta_{k l}$, where $\eta_{k l} \geq 0$ is assigned to arc $(k, l)$ in the construction graph. The attractiveness values may depend on the partial walk $u=$ $\left(u_{0}, \ldots, u_{t-2}, u_{t-1}=k\right)$ the current agent has already traversed, so they can be written as $\eta_{k l}=\eta_{k l}(u)$. Typically, the value $\eta_{k l}(u)$ is obtained from a Greedy Heuristic (GH) for the combinatorial optimization problem under consideration; in this view, it may also be interpreted as the value of a so-called greedy function (see [9]): Assume that a GH is given. It specifies the stepwise construction of a "good" (but in general not optimal) solution of the problem. In our formalism, this construction can be represented by a walk in the construction graph. The GH defines "weights" for all feasible arcs $(k, l)$ leaving node $k$, and determines the next node $l$ of the walk by the "greedy principle" that the weight of $(k, l)$ is maximum. We may now consider the weight of arc $(k, l)$ as the "attractiveness" of the transition from node $k$ to node $l$, that is, we may set $\eta_{k l}(u)=$ weight $(k, l)$. Alternatively, a second way of defining the attractiveness values may also be chosen: Set $\eta_{k l}(u)=1$, if weight $(k, l)$ is maximum among all successor nodes of node $k$, and $\eta_{k l}(u)=0$ otherwise. The reader should notice that the weight of $(k, l)$ is allowed to depend on the whole "history" $u=\left(u_{0}, \ldots, u_{t-2}, u_{t-1}=k\right)$.

The values $\eta_{k l}(u)$ can also be used for locking walks corresponding to infeasible solutions: If $w$ is such a walk, let $u$ be the longest partial walk (starting at the start node) on $w$ that can still be prolonged to a feasible walk (i.e, that did not contradict a feasibility condition so far), and let $(k, l)$ be the first arc on $w$ not belonging to $u$. Then $w$ can be "locked" (without locking any feasible walk) by setting $\eta_{k l}(u)=0$.

In our formalism presented above, GBAS is a natural stochastic generalization of a GH: Note that if the parameter $\alpha$ is set equal to zero, and the second way described above of defining the attractiveness values is chosen, the behavior of the agents is exclusively governed by the greedy principle, so GH is another special case of GBAS.

A mathematically more sophisticated approach covered by the presented formalism consists in deriving the attractiveness $\eta_{k l}(u)$ from a lower bound for the cost values in the subset of the solution space defined by $u$, as it is done in the demanding, but often very efficient ANTS technique developed by Maniezzo [17]. 
It should be emphasized that GBAS is applicable to all combinatorial optimization problems with a finite solution space per problem instance. This point is discussed in detail in [13].

Let us now continue the discussion of section 1. If convergence of the walks of the agents to one optimal walk would hinge on the uniqueness of the optimal walk in an essential way, this would have undesirable consequences. For problems where the exact optimal solution is not unique, there would be no reason to believe that GBAS leads, on the long run, to a situation where only the arcs of the optimal walks have trail levels essentially larger than zero. But even if this should be the case in a particular application, GBAS might nevertheless output suboptimal solutions. To illustrate this important point, we refer to Fig. 2.1. Suppose there are two optimal walks in this construction graph, $w_{1}^{*}=(0,1,3,4,6,7,9,10,12)$ and $w_{2}^{*}=$ $(0,2,3,5,6,8,9,11,12)$, and that the other walks are suboptimal (say, with equally high cost values). Assume that $\eta_{k l}(u)=1$ for all arcs. If the relative frequencies of the traversals of $w_{1}^{*}$ and $w_{2}^{*}$ should tend to the same value $1 / 2$ (which might be conjectured in view of the symmetry and a possible influence of the law of large numbers), all arcs would obtain (nearly) the same trail level. Then, however, also each of the 14 suboptimal walks would have the same probability of being traversed by an agent in the current cycle as $w_{1}^{*}$ or $w_{2}^{*}$. In other words: With a high probability, GBAS would output a suboptimal solution, even if only optimal walks have been reinforced.

Our Theorem 3.1 in the following section implies that, fortunately, this is not the final development to be expected: In the situation described above (and on the conditions of the Theorem), only one of the walks $w_{1}^{*}$ and $w_{2}^{*}$ will survive; the trail levels of the other one will vanish. Showing a general result of this type is especially difficult because of the possibility of overlapping optimal walks: in the case of walks sharing arcs, hardly controllable phenomena may occur, e.g., a rewarded walk may profit less by the trail level update than a not rewarded walk. A rather subtle analysis (using the introduction of a suitable "impact value") will be necessary in order to cope with this problem.

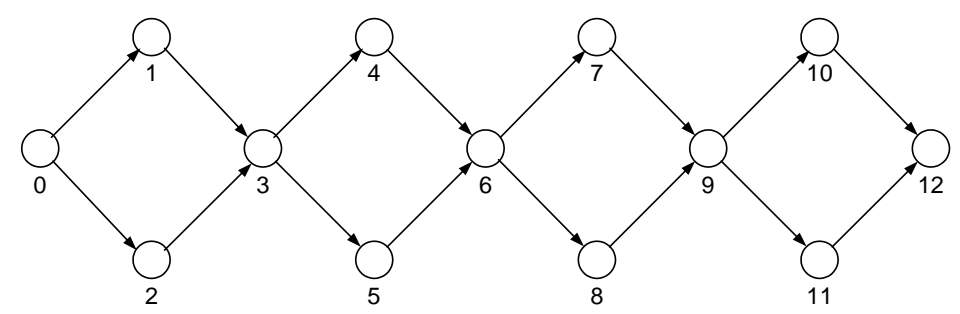

Fig. 2.1. Example construction graph.

\section{The Convergence Result}

The aim of this section is to show that under weaker conditions than those in [13], the current solutions of GBAS (the solutions corresponding to the walks of agent $A_{1}, \ldots, A_{S}$ in cycle $n$ ) converge with a probability that can be made arbitrarily close to unity to one optimal solution. The conditions are the following:

(i) The parameter $\alpha$ in (1) is chosen as $\alpha=1$. 
(ii) There is at least one reachable optimal walk $w^{*}$, that is, an optimal walk $w^{*}$ with the property that the attractiveness values satisfy $\eta_{k l}(u)>0$ for all arcs $(k, l)$ of $w^{*}$ and the corresponding partial walks $u$ of $w^{*}$.

(iii) The reward functions $\varphi_{s}$ chosen for the definition of the values $\Delta \tau_{k l}^{(s)}$ at the beginning of cycle $n+1$ (see eq. (3)) have the following property: Let $f^{*}=f^{*}(n)$ be the lowest cost value observed in the cycles $1, \ldots, n-1$, that is, the lowest objective function value $f_{s}$ corresponding to a walk of an agent $A_{s}$ in these $n-1$ cycles. (It is easy to store and update the current value of $f^{*}$ during the execution of the algorithm.) In the case of cycle $n=1$, let $f^{*}=\infty$. Then for all $n$ larger or equal to some fixed $m_{0} \in \mathbb{N}$,

(i) $\varphi_{s}\left(f_{1}, \ldots, f_{S}\right)>0$ for $f_{s} \leq f^{*}$,

(ii) $\varphi_{s}\left(f_{1}, \ldots, f_{S}\right)=0$ for $f_{s}>f^{*}$.

In other words: After cycle $m_{0}$, only walks that are at least as good as the best found walk up to now get a positive increment $\Delta \tau_{k l}^{(s)}$. For positive cost functions, e.g., $\varphi_{s}\left(f_{0}, \ldots, f_{S}\right)$ may be chosen as $\varphi_{s}\left(f_{0}, \ldots, f_{S}\right)=1 / f_{s}$ if $f_{s} \leq f^{*}$ and $\varphi_{s}\left(f_{0}, \ldots, f_{S}\right)=0$ otherwise. Note that $\varphi_{s}$ is allowed to depend on the "history" (the cycles $1, \ldots, n-1$ ), and hence in particular on the value $f^{*}$.

Condition (i) is identical to condition (a) in [13]. As stated there, this condition does not imply an essential restriction, since the main purpose of the parameters $\alpha$ and $\beta$ is not to "shape" trail levels and attractiveness values (this could be done by other means), but to have easy control on their impact relative to each other; so we may fix one of these two parameters as long as the other remains free.

Condition (ii) is a weaker form of condition (c) in [13] (condition (b) in [13] is the uniqueness condition which we can drop in the present paper). The reader should note that (ii) can easily be satisfied by arbitrarily slight changes of the attractiveness values: for each feasible continuation $(k, l)$ of a partial walk $u$ with $\eta_{k l}(u)=0$, replace $\eta_{k l}(u)=0$ by $\eta_{k l}(u)=\delta$ with a small $\delta>0$. Let us remark that as long as global optimization is intended, it makes sense anyway not to exclude any feasible continuation of a partial walk a priori. - Although not very restrictive, condition (ii) is essential since otherwise it may happen that a specific arc belonging to the optimal walk is inaccessible for the agents, just because of an unlucky choice of the attractiveness values.

Condition (iii), finally, relaxes condition (d) in [13]. It says that after a certain cycle $m_{0}$ (in [13], $m_{0}=1$ was required), a rewarding strategy similar to "Global-best" (see Gambardella and Dorigo [11]) is followed: Only the best walks up to now are rewarded; walks that are dominated by another already traversed walk do not get trail level increments anymore.

Our main result is the following:

Theorem 3.1. Let conditions (i) - (iii) be satisfied, and let $P_{w}(n)$ denote the probability, computed from (1), that a fixed agent $A_{s}$ traverses the walk $w$ in cycle $n$. Then the following two assertions are valid:

1. For each $\epsilon>0$ and for fixed parameters $\rho$ and $\beta$, it can be achieved by the choice of a sufficiently large number $S$ of agents that for an integer $N(\epsilon)$, with a probability larger or equal to $1-\epsilon$, there is an optimal walk $w^{*}$ such that $P_{w^{*}}(n) \geq 1-\epsilon$ for all $n \geq N(\epsilon)$. 
2. For each $\epsilon>0$ and for fixed parameters $S$ and $\beta$, it can be achieved by the choice of an evaporation factor $\rho$ sufficiently close to zero that for an integer $N(\epsilon)$, with a probability larger or equal to $1-\epsilon$, there is an optimal walk $w^{*}$ such that $P_{w^{*}}(n) \geq 1-\epsilon$ for all $n \geq N(\epsilon)$.

The assertion of Theorem 3.1 only makes sense if the values $P_{w}(n)$ are considered as random variables themselves (instead of deterministic probabilities of random events). In order to fix a probability space where the values $P_{w}(n)$ can be given this interpretation, we represent the process induced by the GBAS algorithm in terms of a stochastic process in discrete time, the final part of which (the cycles $m_{0}, m_{1}, \ldots$ ) will turn out as a Markov process (see, e.g., [10]). The states of the stochastic process are the triples

$$
\left(\underline{\tau}(n), \underline{w}(n), f^{*}(n)\right) \quad(n=1,2, \ldots),
$$

where

- $\underline{\tau}(n)$ is the vector of the trail levels $\tau_{k l}(n)$ for all arcs $(k, l)$ during cycle $n$,

- $\underline{w}(n)$ is the vector of the walks $w^{(s)}(n)(s=1, \ldots, S)$ of the agents $A_{1}, \ldots, A_{S}$ in cycle $n$,

- $f^{*}(n)$ is the best found cost value corresponding to the walk of any agent in one of the cycles $1, \ldots, n-1$. For cycle $n=1$, we set $f^{*}(1)=\infty$.

Proposition 3.1. For $n=m_{0}, m_{0}+1, \ldots$, the state variables $\left(\underline{\tau}(n), \underline{w}(n), f^{*}(n)\right)$ form a Markov process.

The proof is a repetition of the proof of Proposition 4.1 in [13], applied to the cycles $m_{0}, m_{0}+$ $1, \ldots$

Remark 3.1. In the considered stochastic process, the numbers $p_{k l}(n, u)$ defined by (1) are functions of $\underline{\tau}(n)$ and therefore also functions of the states of the process in the cycles $1, \ldots, n-1$ (respectivly, only of cycle $n-1$, if $\left.n>m_{0}\right)$. In particular, the numbers $p_{k l}(n, u)$ are random variables. From this point of view, their interpretation as probabilities is only an indirect one: by the state transition rule of the considered stochastic process, they determine the distribution of the vector $\underline{w}(n)$ of the walks in cycle $n$.

In extension of [13], the following abbreviations shall be used:

- $W^{*}=\left\{w_{1}^{*}, \ldots, w_{R}^{*}\right\}$ denotes the set of reachable optimal walks in the sense of condition (ii) above.

- $L(w)$ denotes the length (number of arcs) of walk $w$.

- IP is written for the probability measure on the stochastic process defined above.

- $E_{n}^{(s)}(w)$ denotes the event that $w^{(s)}(n)=w$, that is, the event that agent $A_{s}$ traverses walk $w$ in cycle $n$.

- $B_{n}$ is the event that $w^{(s)}(n) \notin W^{*}$ for all $s=1, \ldots, S$ (the event that no agent traverses an optimal walk in cycle $n$ ). 
- $F_{n}$ is an abbreviation for $B_{1} \wedge \ldots \wedge B_{n-1} \wedge \neg B_{n}$, that is, for the event that at least one optimal walk is traversed by at least one agent in cycle $n$, but by no agent in the cycles $1, \ldots, n-1$. Obviously, the events $F_{1}, F_{2}, \ldots$ are mutually exclusive.

- $A$ is an abbreviation for $F_{1} \vee F_{2} \vee \ldots$, that is, for the event that there is an $n$ and an $s$ such that $w^{(s)}(n) \in W^{*}$ (the event that at least one optimal walk is traversed by at least one agent in some cycle).

Moreover, the notation $(k, l) \in w$ shall be used for the assertion that arc $(k, l)$ lies on the walk $w$, and a walk $w$ shall sometimes also be denoted by the sequence of nodes lying on it.

Because of the definition of $W^{*}$ and the fact that there are only finitely many arcs $(k, l)$ and only finitely many partial walks $u$, we have

$$
\gamma=\min \left\{\left[\eta_{k l}(u)\right]^{\beta} \mid w^{*} \in W^{*},(k, l) \in w^{*}, u \text { partial walk on } w^{*}\right\}>0
$$

and

$$
\Gamma=\max \left\{\left[\eta_{k l}(u)\right]^{\beta} \mid(k, l) \text { arc, } u \text { partial walk }\right\}<\infty .
$$

Multiplying all attractiveness values $\eta_{k l}(u)$ by a fixed constant does not change the transition probabilities (1). So it can be assumed without loss of generality that the values $\eta_{k l}(u)$ are normalized in such a way that $\Gamma=1$, that is,

$$
\left[\eta_{k l}(u)\right]^{\beta} \leq 1
$$

for all $\operatorname{arcs}(k, l)$ and all partial walks $u$.

The following Lemma 3.1 is an extension of Lemma 4.1 in [13].

Lemma 3.1. For each $n \geq 1$, the conditional probability, given arbitrary events in cycle $1, \ldots, n-1$, that event $\neg B_{n}$ occurs, is larger or equal to $1-\left(1-c^{n-1} p\right)^{S}$, where $c=(1-\rho)^{L_{0}}$, $p=(\gamma /|\mathcal{A}|)^{L_{0}}$, and $L_{0}=\min \left\{L\left(w^{*}\right) \mid w^{*} \in W^{*}\right\}$.

Proof. Let $w^{*} \in W^{*}$ be a fixed walk of length $L_{0}$. We want to determine a lower bound for the probability that in cycle $n$, there is at least one agent who traverses $w^{*}$, given arbitrary events in the cycles before. Since $\Delta \tau_{k l} \geq 0$ and $\rho>0$, eq. (5) implies

$$
\tau_{k l}(n+1) \geq(1-\rho) \tau_{k l}(n)
$$

in the case $C>0$, and again because of $\rho>0$, this holds also in the case $C=0$. Repeated application of (9) yields

$$
\tau_{k l}(n) \geq(1-\rho)^{n-1} \tau_{k l}(1) .
$$

This estimate holds independently of the events in previous cycles. Because of (8) and $\sum_{(k, l)} \tau_{k l}(n)=1$,

$$
\sum_{r \notin u,(k, r) \in \mathcal{A}} \tau_{k r}(n) \cdot\left[\eta_{k r}(u)\right]^{\beta} \leq \sum_{r \notin u,(k, r) \in \mathcal{A}} \tau_{k r}(n) \leq 1 .
$$

Therefore, the transition probabilities $p_{k l}(n, u)$ given by (1) satisfy, for a node $l$ with $l \notin u$, the inequality

$$
p_{k l}(n, u)=\frac{\tau_{k l}(n)\left[\eta_{k l}(u)\right]^{\beta}}{\sum_{r \notin u,(k, r) \in \mathcal{A}} \tau_{k r}(n)\left[\eta_{k r}(u)\right]^{\beta}} \geq \tau_{k l}(n)\left[\eta_{k l}(u)\right]^{\beta} .
$$


Let $w^{*}=\left(v_{0}, \ldots, v_{L_{0}}\right)$. Then by $(11),(7)$ and $(10)$,

$$
\begin{aligned}
& \mathbb{P}\left(E_{n}^{(s)}\left(w^{*}\right)\right)=\prod_{i=0}^{L_{0}-1} p_{v_{i} v_{i+1}}\left(n,\left(v_{0}, \ldots, v_{i}\right)\right) \geq \prod_{i=0}^{L_{0}-1} \tau_{v_{i} v_{i+1}}(n)\left[\eta_{v_{i} v_{i+1}}\left(v_{0}, \ldots, v_{i}\right)\right]^{\beta} \\
& \geq \gamma^{L_{0}} \prod_{i=0}^{L_{0}-1} \tau_{v_{i} v_{i+1}}(n) \geq \gamma^{L_{0}} \prod_{i=0}^{L_{0}-1}(1-\rho)^{n-1} \tau_{v_{i} v_{i+1}}(1) \\
&=\gamma^{L_{0}}(1-\rho)^{L_{0}(n-1)} \prod_{(k, l) \in w^{*}} \tau_{k l}(1)=\gamma^{L_{0}}(1-\rho)^{L_{0}(n-1)} \cdot(1 /|\mathcal{A}|)^{L_{0}}=c^{n-1} p .
\end{aligned}
$$

Since the walks of the $S$ agents are independent, this implies

$$
\mathbb{P}\left(B_{n}\right) \leq \mathbb{P}\left(\neg E_{n}^{(1)}\left(w^{*}\right) \wedge \ldots \wedge \neg E_{n}^{(S)}\left(w^{*}\right)\right) \leq\left(1-c^{n-1} p\right)^{S},
$$

which yields the assertion.

In the following lemmas, we consider the stochastic process defined above conditioned on the event $F_{m}$, that is, on the event that cycle $m$ is the first cycle where an optimal walk is traversed. The conditional probability of an event, given $F_{m}$, will be denoted in the usual way by $\mathbb{P}\left\{\right.$ event $\left.\mid F_{m}\right\}$. For the formulation of the lemmas, it is convenient to introduce some additional definitions:

Definition 3.1. We distinguish two possible cycle types: cycle $n$ is called a $B$-cycle, if event $B_{n}$ occurs, that is, of no agent traverses an optimal walk in cycle $n$, and it is called a $\neg B$-cycle, if event $\neg B_{n}$ occurs, that is, if at least some agent traverses an optimal walk in cycle $n$.

An epoch is a sequence of consecutive cycles after cycle $m_{1}=\max \left(m_{0}, m\right)$ (cycle $m$ being the first $\neg B$-cycle) with the property that all these consecutive cycles are $B$-cycles, except the last, which is a $\neg B$-cycle.

A period is a sequence of a fixed number of consecutive epochs. (The number of epochs contained in a period will be determined later.)

During a fixed epoch, the trail levels remain constant, since at the end of a $B$-cycle after cycle $m_{1}=\max \left(m_{0}, m\right)$, the value $C$ is set equal to zero in view of condition (iii), so no change takes place. Only after the last cycle of an epoch, the trail levels change. Therefore, we may consider the cycles of an epoch as independent trials (with identical success probabilities) to produce the event $\neg B_{n}$. As soon as $\neg B_{n}$ occurs, the epoch ends. The distribution of the states during an epoch depends on previous epochs only via the trail levels at the beginning of the epoch. Moreover, the probability of any event in the last cycle of an epoch is identical to the conditional probability of this event in a fixed cycle $n$ of the current epoch (that is, in a cycle with the trail levels valid in this epoch), given the event $\neg B_{n}$.

Definition 3.2. The relative trail level $\psi_{k l}(n)$ of an arc $(k, l)$ is defined by

$$
\psi_{k l}(n)=\frac{\tau_{k l}(n)}{\sum_{(k, r) \in \mathcal{A}} \tau_{k r}(n)} .
$$

Note that the sum is here over all $\operatorname{arcs}(k, r)$ with $k$ as the start node, including those with $r \in u$. 
In view of $(1),(7)$ and $(8)$, for each $\operatorname{arc}(k, l)$ on a walk $w^{*} \in W^{*}$ with partial walk $u_{w^{*}}(k)$ up to node $k$,

$$
p_{k l}\left(n, u_{w^{*}}(k)\right) \geq \frac{\tau_{k l}(n) \cdot \gamma}{\sum_{r \notin u,(k, r) \in \mathcal{A}} \tau_{k r}(n) \cdot 1} \geq \gamma \frac{\tau_{k l}(n)}{\sum_{(k, r) \in \mathcal{A}} \tau_{k r}(n)}=\gamma \psi_{k l}(n) .
$$

Definition 3.3. The impact value $\Psi\left(w^{*}\right)$ of a walk $w^{*}$ is the product of the relative trail levels of its arcs:

$$
\Psi\left(w^{*}\right)=\prod_{(k, l) \in w^{*}} \psi_{k l}(n)
$$

As a consequence of (12), we get the following relation between the probability of a walk $w^{*} \in W^{*}$ to be traversed, and its impact value:

$$
P_{w^{*}}(n)=\prod_{(k, l) \in w^{*}} p_{k l}\left(n, u_{w^{*}}(k)\right) \geq \gamma^{L\left(w^{*}\right)} \Psi\left(w^{*}\right)
$$

for each $w^{*} \in W^{*}$.

For $n \geq m_{1}=\max \left(m_{0}, m\right)$, it will be convenient to consider, in parallel to the trail level update mechanism (5), a slightly different alternative way of updating the trail levels, defined by

$$
\begin{gathered}
\tilde{\tau}_{k l}\left(m_{1}\right)=\tau_{k l}\left(m_{1}\right), \\
\tilde{\tau_{k l}}(n+1)=\tilde{\tau}_{k l}(n)+\frac{\rho}{1-\rho} \Delta \tau_{k l} \quad\left(n \geq m_{1}\right) .
\end{gathered}
$$

The r.h.s. of (14) is equal to the trail level that would have been obtained by the original update rule, multiplied by the factor $1 /(1-\rho)$. Furthermore, if an arc $(k, l)$ is not rewarded (that is, if $\left.\Delta \tau_{k l}=0\right)$, then its trail level remains now constant instead of being subject to evaporation, so we may call this variant evaporation-less trail level update. It is clear that the sum of trail levels computed in this way is not equal to unity anymore; instead, it is multiplied by the factor $1 /(1-\rho)$ in each $\neg B$-cycle. However, the computation of the probabilities $p_{k l}(n, u)$ can be based as well on the values $\tilde{\tau}_{k l}(n)$ instead of the values $\tau_{k l}(n)$, yielding the same results, since, by the denominator of $(1)$, the factor $1 /(1-\rho)$ cancels out. So, both alternatives of the trail level update are equivalent. Also the computation of the relative trail level $\psi_{k l}(n)$ and of the impact value $\Psi\left(w^{*}\right)$ can be based on the values $\tilde{\tau}_{k l}(n)$ instead of the values $\tau_{k l}(n)$ with the same result.

We call the numbers $\tilde{\tau}_{k l}(n)$ the gross trail levels. With their help, Definition 3.4 below which is crucial for our proof can be given:

Let us label the epochs after cycle $m_{1}$ by $h=1,2, \ldots$. In epoch $h$, walk $w_{j}^{*} \in W^{*}(1 \leq j \leq R)$ obtains a certain nonnegative gross level increment $\pi_{j}(h)$ for each of its arcs according to the evaporation-less trail level update rule described above. Note that this increment is the same for each arc of $w_{j}^{*}$, but, of course, an arc $(k, l)$ can possibly obtain such increments from different (rewarded) walks on which it lies. Now let

$$
\mathcal{I}_{j}(h)=\sum_{h^{\prime}=1}^{h} \pi_{j}\left(h^{\prime}\right)
$$


be the total gross level increment an arc of $w_{j}^{*}$ has obtained in the epochs $1, \ldots, h$ due to the rewards for walk $w_{j}^{*}$. (If the arc belongs also to other walks on $W^{*}$, it may have obtained additional total gross level increments from these other walks. Walks not contained in $W^{*}$, however, are not rewarded anymore after cycle $m_{1}$.)

Definition 3.4. By the champion of epoch $h$, we understand a walk $w_{\nu}^{*} \in W^{*}$ for which $\mathcal{I}_{\nu}(h)=\max \left\{\mathcal{I}_{j}(h) \mid 1 \leq j \leq R\right\}$. (If there is more than one $w_{j}^{*}$ with $\mathcal{I}_{j}(h)=\max$, the champion may be chosen arbitrarily from these walks.) By the champion of a period, we understand the champion of the first epoch of this period.

It is now possible to show the following basic lower bound estimation:

Lemma 3.2. Conditional on event $F_{m}$, the champion $w_{\nu}^{*}$ of an epoch $h$ after cycle $m_{1}=$ $\max \left(m_{0}, m\right)$ has always a probability $P_{w_{\nu}^{*}}(n) \geq \mu_{0}>0$ to be traversed by a fixed agent $A_{s}$, where the number $\mu_{0}$ only depends on $\gamma, R, \rho, m_{1}$, and the structure of the construction graph.

Proof. We start the proof with the following observation: Let $c>0,0<\xi \leq x \leq 1,0 \leq y \leq 1$, and

$$
f(a)=\frac{x+a}{x+y+c a} \quad(a \geq 0) .
$$

Then there is a number $\mu=\mu(c, \xi)>0$ such that $f(a) \geq \mu$ for all $a \geq 0$. This is easily verified by distinguishing the two cases $a \leq 2 / c$ and $a>2 / c$ : In the first case, we obtain $f(a) \geq \xi / 4$, whereas in the second case, $f(a) \geq 1 /(2 c)$. So the assertion holds with

$$
\mu=\min \left(\frac{\xi}{4}, \frac{1}{2 c}\right) .
$$

Now consider a fixed arc $(k, l)$ contained in the champion $w_{\nu}^{*}$ and its "sister $\operatorname{arcs"}(k, r)$ sharing with $(k, l)$ the start node $k$. Let $x_{k l}$ and $x_{k r}$ be the gross trail levels of $(k, l)$ and $(k, r)$, respectively, in cycle $m_{1}$. Let $a=\mathcal{I}_{\nu}(h)$. In epoch $h$, the gross trail level of $(k, l)$ has increased at least to the value $x_{k l}+a$ and at most to the value $x_{k l}+R a$, compared to cycle $m_{1}$. (Note that $(k, l)$ may be contained in several optimal - and therefore possibly rewarded - walks.) The gross trail level of an arc $(k, r)(r \neq l)$, however, has increased at most to the value $x_{k r}+(R-1) a$, since $\mathcal{I}_{j}(h) \leq a$ for all $j \neq \nu$, and $(k, r) \notin w_{\nu}^{*}$.

Denoting by $d$ the maximal outdegree of a node in the construction graph, we conclude that the relative trail level (which is identical to the relative gross trail level) of arc $(k, l)$ in epoch $h$ is larger or equal to

$$
\frac{x_{k l}+a}{x_{k l}+R a+\sum_{r} x_{k r}+(d-1)(R-1) a} \geq \frac{x_{k l}+a}{x_{k l}+\sum_{r} x_{k r}+d R a},
$$

where the sum $\sum_{r} x_{k r}$ is over all "sister $\operatorname{arcs"}(k, r)$ of $(k, l)$. Hence the impact value $\Psi\left(w_{\nu}^{*}\right)$ of the champion $w_{\nu}^{*}$ in epoch $h$ is larger or equal to

$$
\prod_{(k, l) \in w_{\nu}^{*}} \frac{x_{k l}+a}{x_{k l}+\sum_{r} x_{k r}+d R a} .
$$

Applying the observation at the beginning of the proof with $x=x_{k l} \in[\xi, 1]$ ( $\xi$ will be determined later), $y=\sum_{r} x_{k r} \in[0,1]$, and $c=d R>0$, yields the existence of a number $\mu>0$, depending 
only on $d, R$, and a lower bound $\xi=\xi\left(m_{1}\right)>0$ of the (gross) trail levels in cycle $m_{1}$, such that the impact value $\Psi\left(w_{\nu}^{*}\right)$ of the champion is bounded below by $\mu^{L\left(w_{\nu}^{*}\right)}$. (Let us emphasize that $\mu$ is independent of the epoch; which walk is the champion, however, depends on the epoch.) By (15), $\mu<1$, and hence by (13),

$$
P_{w_{\nu}^{*}}(n) \geq \gamma^{L\left(w_{\nu}^{*}\right)} \cdot \Psi\left(w_{\nu}^{*}\right) \geq \gamma^{L\left(w_{\nu}^{*}\right)} \cdot \mu^{L\left(w_{\nu}^{*}\right)} \geq \gamma^{L} \mu^{L}
$$

with

$$
L=\max \left\{L\left(w^{*}\right) \mid w^{*} \in W^{*}\right\} .
$$

All that remains to show is: A lower bound $\xi=\xi\left(m_{1}\right)>0$, depending only on $\rho, m_{1}$, and the structure of the construction graph, can be found for the (gross) trail levels in cycle $m_{1}$. This, however, follows by eq. (10) from

$$
\tau_{k l}\left(m_{1}\right) \geq(1-\rho)^{m_{1}-1} \tau_{k l}(1)=(1-\rho)^{m_{1}-1} /|\mathcal{A}| .
$$

Corollary. Conditional on event $F_{m}$, the probability that in the final cycle of a fixed epoch after cycle $m_{1}=\max \left(m_{0}, m\right)$, the champion $w_{\nu}^{*}$, but no other optimal walk is traversed by an agent, is larger or equal to $\mu_{0}^{S}$ (with $\mu_{0}$ from Lemma 3.2). This holds independently of arbitrary events in epochs before the considered epoch.

Proof. Since an epoch consists of independent trials until event $\neg B_{n}$ occurs, the probability we ask for is

$$
\begin{gathered}
\mathbb{P}\left\{\text { no agent traverses a walk } w^{*} \in W^{*} \backslash\left\{w_{\nu}^{*}\right\} \mid \neg B_{n} \wedge F_{m}\right\} \\
\geq \mathbb{P}\left\{\text { no agent traverses a walk } w^{*} \in W^{*} \backslash\left\{w_{\nu}^{*}\right\} \wedge \neg B_{n} \mid F_{m}\right\} \\
\geq \mathbb{P}\left\{\text { all agents traverse } w_{\nu}^{*} \mid F_{m}\right\}=\left[P_{w_{\nu}^{*}}(n)\right]^{S} \geq \mu_{0}^{S} .
\end{gathered}
$$

If we condition on certain events in previous epochs, these events can enter into the probability computation only via the trail levels at the beginning of the given epoch. The estimation above, however, holds independently of the specific values of these trail levels.

Remark 3.2. From the definition of the champion of an epoch, it follows that if $w_{\nu}^{*}$ is the champion of epoch $h$, and $w_{\nu}^{*}$ is the only optimal walk traversed by an agent in epoch $h$, then $w_{\nu}^{*}$ is also the champion of epoch $h+1$.

Remark 3.2 implies that whenever the champion $w_{\nu}^{*}$ of a period (cf. Definition 3.4) is the only traversed walk in all epochs of this period, then $w_{\nu}^{*}$ is also the champion of each epoch of the considered period. Then, by Lemma 3.2, in each cycle $n$ of this period, the variable $P_{w_{\nu}^{*}}(n)$ is larger or equal to $\mu_{0}$.

Lemma 3.3. For a fixed $n \geq m_{1}$, let $w^{*} \in W^{*}, p_{1}=P_{w^{*}}(n)$, and

$$
\bar{p}_{2}=\sum_{w \in W^{*} \backslash\left\{w^{*}\right\}} P_{w}(n) .
$$

Then if $p_{1} \geq 1 / 2$, the probability that in the final cycle of the epoch containing cycle $n$, walk $w^{*}$ is the only walk traversed by an agent, is larger or equal to $1-2 S \bar{p}_{2}$. 
Proof. The considered probability is

$$
\begin{aligned}
& \mathbb{P}\left\{\text { no agent traverses a walk } w \in W^{*} \backslash\left\{w^{*}\right\} \mid \neg B_{n} \wedge F_{m}\right\} \\
= & \frac{\mathbb{P}\left\{\text { no agent traverses a walk } w \in W^{*} \backslash\left\{w^{*}\right\} \wedge \neg B_{n} \mid F_{m}\right\}}{\operatorname{PP}\left(\neg B_{n}\right)} .
\end{aligned}
$$

The denominator of the last expression is equal to $1-\mathbb{P}\left(B_{n}\right)=1-\left(1-p_{1}-\bar{p}_{2}\right)^{S}$. By elementary probabilistic calculations, one finds that the nominator of the expression is equal to

$$
\left(1-\bar{p}_{2}\right)^{S}\left[1-\left(1-\frac{p_{1}}{1-\bar{p}_{2}}\right)^{S}\right]=\left(1-\bar{p}_{2}\right)^{S}-\left(1-p_{1}-\bar{p}_{2}\right)^{S} .
$$

So the probability that in the final cycle, $w^{*}$ is not the only traversed walk, is

$$
\begin{aligned}
1-\frac{\left(1-\bar{p}_{2}\right)^{S}-\left(1-p_{1}-\bar{p}_{2}\right)^{S}}{1-\left(1-p_{1}-\bar{p}_{2}\right)^{S}} & =\frac{1-\left(1-\bar{p}_{2}\right)^{S}}{1-\left(1-p_{1}-\bar{p}_{2}\right)^{S}} \\
\leq 2\left[1-\left(1-\bar{p}_{2}\right)^{S}\right] & \leq 2 S \bar{p}_{2}
\end{aligned}
$$

since $1-p_{1}-\bar{p}_{2} \leq 1 / 2$ for $p_{1} \geq 1 / 2$, and $(1-x)^{n} \geq 1-n x$ for $0<x<1$.

Now consider a fixed period. Let $w_{\nu}^{*}$ be the champion of this period (that is, the champion of its first epoch). For abbreviation, we use the following notation:

- $H^{[k]}$ is the event that in the $k$ th epoch of the given period, walk $w_{\nu}^{*}$ is the only traversed optimal walk,

- $C^{[k]}$ is the event that $w_{\nu}^{*}$ is the champion of the $k$ th epoch of the given period.

Lemma 3.4. For a fixed period after cycle $m_{1}$ and for each $K \in \mathbb{N}$,

$$
\mathbb{P}\left(H^{[1]} \wedge \ldots \wedge H^{[K]} \mid C^{[1]}\right) \geq \mu_{0}^{S K}
$$

with the number $\mu_{0}$ of Lemma 3.2.

Proof. The considered probability is equal to

$$
\mathbb{P}\left(H^{[1]} \mid C^{[1]}\right) \cdot \mathbb{P}\left(H^{[2]} \mid H^{[1]} \wedge C^{[1]}\right) \cdot \ldots \cdot \mathbb{P}\left(H^{[K]} \mid H^{[1]} \wedge \cdots \wedge H^{[K-1]} \wedge C^{[1]}\right) .
$$

Remark 3.2 states that $C^{[k]} \wedge H^{[k]} \Rightarrow C^{[k+1]}$. By repeated application, we obtain

$$
H^{[1]} \wedge \cdots \wedge H^{[k-1]} \wedge C^{[1]} \Rightarrow C^{[k]},
$$

so

$$
H^{[1]} \wedge \cdots \wedge H^{[k-1]} \wedge C^{[1]} \wedge C^{[k]} \Leftrightarrow H^{[1]} \wedge \cdots \wedge H^{[k-1]} \wedge C^{[1]} .
$$

Therefore, by the Corollary to Lemma 3.2,

$$
\mathbb{P}\left(H^{[k]} \mid H^{[1]} \wedge \ldots \wedge H^{[k-1]} \wedge C^{[1]}\right)=\mathbb{P}\left(H^{[k]} \mid\left(H^{[1]} \wedge \ldots \wedge H^{[k-1]} \wedge C^{[1]}\right) \wedge C^{[k]}\right) \geq \mu_{0}^{S},
$$

which completes the proof. 
Lemma 3.5. Let $\mathcal{T}\left(n, w^{*}, \epsilon\right)$ denote the assertion

$$
\left|\tau_{k l}(n)-\frac{1}{L\left(w^{*}\right)}\right|<\epsilon \quad \forall(k, l) \in w^{*} \quad \wedge \quad \tau_{k l}(n)<L\left(w^{*}\right) \epsilon \quad \forall(k, l) \notin w^{*} .
$$

Then to each $\epsilon>0$ and $m \in \mathbb{N}$,

$$
\mathbb{P}\left\{\exists n \geq m_{1}, w^{*} \in W^{*}: \mathcal{T}\left(n, w^{*}, \epsilon\right) \mid F_{m}\right\} \geq 1-\epsilon
$$

where $m_{1}=\max \left(m_{0}, m\right)$.

Proof. Let $\epsilon>0$ and $m \in \mathbb{N}$ be fixed. We construct $\sigma(\epsilon)$ periods after cycle $m_{1}$, each period consisting of $K(\epsilon)$ epochs. The numbers $\sigma(\epsilon)$ and $K(\epsilon)$ will be determined later. At the beginning of each period, its champion is determined. According to Lemma 3.4, for each considered period, the champion of this period is, with a probability larger or equal to $\mu_{0}^{S K(\epsilon)}$, the only traversed optimal walk in the entire period. This estimation holds independently of what has happened in the previous periods. Again by considering conditional probabilities (as in the proof of Lemma 3.4), one obtains the result that among the $\sigma(\epsilon)$ successive periods, there is with a probability larger or equal to

$$
1-\left[1-\mu_{0}^{S K(\epsilon)}\right]^{\sigma(\epsilon)}
$$

at least one period with the property that its champion is in all $K(\epsilon)$ epochs of this period the only traversed optimal walk. Because of $1-\mu_{0}^{S K(\epsilon)}<1$, it is possible to choose the number $\sigma(\epsilon)$ (to the given $\epsilon, r, S$, and $K(\epsilon)$ ) large enough that

$$
\left[1-\mu_{0}^{S K(\epsilon)}\right]^{\sigma(\epsilon)}<\epsilon
$$

such that the expression (17) gets larger or equal to $1-\epsilon$. In other words: With a probability $\geq 1-\epsilon$ (conditional on $F_{m}$ ), there is a period containing only epochs where one and the same walk, say $w^{*}$, is rewarded.

We show now that at the end of this period,

$$
\left|\tau_{k l}(n)-\frac{1}{L\left(w^{*}\right)}\right|<\epsilon \quad \text { for all }(k, l) \in w^{*}
$$

holds, provided that $K(\epsilon)$ is sufficiently large. It is easy to verify from (4) - (6) (cf. [13], proof of Lemma 4.2) that in the case where only walk $w^{*}$ is rewarded in cycle $n$,

$$
\tau_{k l}(n+1)-\frac{1}{L\left(w^{*}\right)}=(1-\rho)\left(\tau_{k l}(n)-\frac{1}{L\left(w^{*}\right)}\right) \quad \text { for }(k, l) \in w^{*},
$$

that is, the distance between $\tau_{k l}$ and $1 / L\left(w^{*}\right)$ is reduced to a portion of $1-\rho$ of the former distance. Choosing $K(\epsilon)$ large enough that $(1-\rho)^{K(\epsilon)}<\epsilon$ effects therefore a reduction of the distance between $\tau_{k l}(n)$ and $1 / L\left(w^{*}\right)$ to a value smaller or equal to $\epsilon$.

The assertion $\tau_{k l}(n)<L\left(w^{*}\right) \epsilon$ for all $(k, l) \notin w^{*}$ follows immediately since the sum of all trail levels $\tau_{k l}(n)$ is unity. 
Lemma 3.6. Let $L$ be given by (16), and suppose that for some $w^{*} \in W^{*}$ and some $n \in \mathbb{I N}$, we have

$$
\tau_{k l}(n) \geq \frac{1}{2 L} \quad \text { for all }(k, l) \in w^{*}
$$

Furthermore, let

$$
D=2 L d / \gamma
$$

with $d=$ maximal outdegree of a node in the construction graph, and suppose

$$
\tau_{\text {max }}=\max \left\{\tau_{k r}(n) \mid k \in w^{*}, r \notin w^{*}\right\} \leq 1 / D .
$$

Then the probability that a fixed agent traverses some walk different from $w^{*}$ is smaller or equal to $L D \tau_{\max }$.

Proof. By assumption and by definition of $p_{k l}(n, u)$, the probability that a fixed agent located at node $k$ of $w^{*}$ does not proceed to the successor node $l$ of $k$ on $w^{*}$, but to another node $r$, is smaller or equal to

$$
d \cdot \frac{\tau_{\max } \cdot 1}{(1 /(2 L)) \cdot \gamma}=D \tau_{\max }
$$

Hence, the probability that a walk different from $w^{*}$ is traversed, is smaller or equal to

$$
1-\prod_{(k, l) \in w^{*}}\left(1-D \tau_{\max }\right)=1-\left(1-D \tau_{\max }\right)^{L} \leq 1-\left(1-L D \tau_{\max }\right)
$$

because of $(1-x)^{n} \geq 1-n x$ for $0<x<1$.

Lemma 3.7. Let $\mathcal{T}\left(n, w^{*}, \epsilon\right)$ be defined as in Lemma 3.5. Then, for all $\epsilon>0$ and $m \in \mathbb{N}$, there is an $N \in \mathbb{N}$ such that

$$
\mathbb{P}\left\{\exists w^{*} \in W^{*} \forall n^{\prime} \geq N: \mathcal{T}\left(n^{\prime}, w^{*}, \epsilon\right) \mid F_{m}\right\} \geq \epsilon .
$$

Proof. We start by showing that for all $\epsilon>0$ and $m \in \mathbb{N}$,

$$
\mathbb{P}\left\{\exists n \geq m, w^{*} \in W^{*} \forall n^{\prime} \geq n: \mathcal{T}\left(n^{\prime}, w^{*}, \epsilon\right) \mid F_{m}\right\} \geq \epsilon .
$$

First, to the given $\epsilon>0$, a number $\tilde{\epsilon}>0$ is determined as follows: For fixed $\rho$,

$$
\lim _{\delta \rightarrow 0}\left[1-\exp \left(-\frac{2 \delta}{\rho}\right)\right]=0 .
$$

Thus a $\delta>0$ (depending on $\epsilon$ and $\rho$ ) can be found such that $1-\exp (-2 \delta / \rho) \leq \epsilon / 2$. W.l.o.g., let $\delta \leq 1 / 2$. Now for $L$ given by (16) and $D$ given by (19), we set

$$
\tilde{\epsilon}=\min \left\{\frac{\epsilon}{2}, \frac{1}{2 L}, \frac{\delta}{2 S L^{2} D}\right\} .
$$

Replacing $\epsilon$ by $\tilde{\epsilon}$ in Lemma 3.5, we obtain that with a probability (conditional on $F_{m}$ ) larger or equal to $1-\tilde{\epsilon} \geq 1-\epsilon / 2$, a cycle $n \geq m_{1}$ and a walk $w^{*} \in W^{*}$ exist, such that $\mathcal{T}\left(n, w^{*}, \tilde{\epsilon}\right)$ holds, that is, such that

$$
\left|\tau_{k l}(n)-\frac{1}{L\left(w^{*}\right)}\right|<\tilde{\epsilon} \quad \text { for all }(k, l) \in w^{*}
$$


and

$$
\tau_{k l}(n)<L\left(w^{*}\right) \tilde{\epsilon} \quad \text { for all }(k, l) \notin w^{*} .
$$

Let us suppose that $\mathcal{T}\left(n, w^{*}, \tilde{\epsilon}\right)$ holds indeed. Then, in particular,

$$
\tau_{k l}(n) \geq \frac{1}{L\left(w^{*}\right)}-\tilde{\epsilon} \geq \frac{1}{L}-\tilde{\epsilon} \geq \frac{1}{2 L} \quad \text { for all }(k, l) \in w^{*},
$$

and

$$
\tau_{k l}(n) \leq L \tilde{\epsilon} \leq \frac{\delta}{2 S L D}<\frac{1}{D} \quad \text { for all }(k, l) \notin w^{*},
$$

hence the conditions of Lemma 3.6 are satisfied. It follows that

$$
p_{1}=P_{w^{*}}(n) \geq 1-L D \tau_{\max }
$$

So, because of (22),

$$
p_{1} \geq 1-L D \cdot L\left(w^{*}\right) \tilde{\epsilon} \geq 1-L^{2} D \tilde{\epsilon} \geq 1-\frac{\delta}{2 S}>\frac{1}{2} .
$$

Let us now consider the epoch containing cycle $n$ (we call it epoch 0 ), and the epochs after this epoch, which we label by $\lambda=1,2, \ldots$ in this proof. (The reader should be aware of the different labeling in this proof, in Definition 3.4, and in Lemma 3.4.) Similarly as in Lemma 3.4 , let $H$ denote the event that in a considered epoch, walk $w^{*}$ is the only traversed walk. For each epoch $\lambda$, we ask for the conditional probability of the event $H$, given that event $H$ has occurred in all previous epochs $1, \ldots, \lambda-1$. To estimate this probability, we apply each time Lemma 3.6. Since on the condition that $H$ has occurred in all previous epochs, the trail levels of $\operatorname{arcs}(k, l) \notin w^{*}$ have decreased geometrically with factor $1-\rho$, we have

$$
\tau_{\max }^{[\lambda]}=(1-\rho)^{\lambda} \tau_{\max }^{[0]}
$$

where $\tau_{\max }$ is given by (20), and the indices in brackets refer to the epoch numbers. Because, on the other hand, on the condition that $H$ has occurred in all previous epochs, levels on $\operatorname{arcs}(k, l) \in w^{*}$ have approached the value $1 / L\left(w^{*}\right)$ (see $(18)$ ), the condition $\tau_{k l}(n) \geq 1 /(2 L)$ for $(k, l) \in w^{*}$ of Lemma 3.6 remains always satisfied. Moreover, the probability $P_{w^{*}}(n)$ of a traversal of $w^{*}$ cannot decrease at the end of an epoch where event $H$ occurs, as is easily seen by considering the gross trail levels introduced before Definition 3.4, so (23) implies also $p_{1}^{[\lambda]} \geq p_{1}^{[0]}>1 / 2$ for all $\lambda$ (again, the indices in brackets refer to the epoch numbers). Applying Lemma 3.6, we obtain: The probability that a fixed agent traverses some walk different from $w^{*}$ in epoch $\lambda$, given event $H$ has occurred in the epochs $1, \ldots, \lambda-1$, is smaller or equal to

$$
L D \tau_{\max }^{[\lambda]}=L D(1-\rho)^{\lambda} \tau_{\max }^{[0]} .
$$

In particular, the probability $\bar{p}_{2}$ of traversing an optimal walk different from $w^{*}$ in a cycle of epoch $\lambda$ is then smaller or equal to the expression in (24).

Now let us apply Lemma 3.3. As verified above, the condition $p_{1} \geq 1 / 2$ is satisfied. Therefore, using (22), we conclude that the conditional probability $q^{[\lambda]}$ that in the final cycle of epoch $\lambda$, walk $w^{*}$ is not the only traversed walk, given event $H$ has occurred in the previous epochs $1, \ldots, \lambda-1$, is smaller or equal to

$$
2 S \cdot L D(1-\rho)^{\lambda} \tau_{\max }^{[0]} \leq 2 S L D(1-\rho)^{\lambda} \cdot L \tilde{\epsilon}
$$




$$
\leq 2 S L^{2} D(1-\rho)^{\lambda} \cdot \delta /\left(2 S L^{2} D\right)=(1-\rho)^{\lambda} \delta .
$$

As a consequence, the probability that there is an epoch $\lambda$ where the event $\neg H$ occurs, is

$$
1-\prod_{\lambda=1}^{\infty}\left(1-q^{[\lambda]}\right) \leq 1-\prod_{\lambda=1}^{\infty}\left(1-(1-\rho)^{\lambda} \delta\right)
$$

Because of $(1-\rho)^{\lambda} \delta \leq \delta \leq 1 / 2$ and $\log (1-x) \geq-2 x$ for $x \leq 1 / 2$,

$$
\log \prod_{\lambda=1}^{\infty}\left(1-(1-\rho)^{\lambda} \delta\right) \geq-2 \sum_{\lambda=1}^{\infty}(1-\rho)^{\lambda} \delta \geq-\frac{2 \delta}{\rho}
$$

and therefore

$$
1-\prod_{\lambda=1}^{\infty}\left(1-q^{[\lambda]}\right) \leq 1-\exp \left(-\frac{2 \delta}{\rho}\right) \leq \frac{\epsilon}{2} .
$$

Thus, conditional on $\mathcal{T}\left(n, w^{*}, \tilde{\epsilon}\right)$, with a probability of at least $1-\epsilon / 2$, event $H$ occurs in all epochs $1,2, \ldots$ As a consequence of Lemma 3.5, the probability (conditional on $F_{m}$ ) for the existence of an $n$ with $\mathcal{T}\left(n, w^{*}, \epsilon\right)$ is larger or equal to $1-\tilde{\epsilon} \geq 1-\epsilon / 2$. Therefore, the probability for $\mathcal{T}\left(n, w^{*}, \epsilon\right)$ and event $H$ in all epochs following cycle $n$ is larger or equal to $(1-\epsilon / 2)^{2} \geq 1-\epsilon$, which proves assertion (21), since the occurrence of $H$ in an epoch leaves $\mathcal{T}\left(n, w^{*}, \tilde{\epsilon}\right)$ satisfied after the current trail level update.

In the formulation (21), the number $n$ still depends on the random influence, which we may denote by $\omega$. However, in order to prove the Lemma, we would need $n$ outside of $\mathbb{P}$, such that it depends on $\epsilon$ and $m$, but not on $\omega$. This can be achieved as follows: Let us always assume that $F_{m}$ holds. According to the proof of Lemma 3.5, $n$ is bounded above by the total number $Z$ of cycles between the first cycle and the end of the considered sequence of $\sigma(\epsilon)$ periods, each consisting of $K(\epsilon)$ epochs. $Z$ is almost surely finite, since the probability of the occurrence of a $\neg B$-cycle is larger than some strictly positive constant by Lemma 3.2. Thus, $Z$ can be considered as a finite discrete random variable (whose distribution, of course, depends on $m$ ). Consequently, for any given $\epsilon>0$ and $m \in \mathbb{N}$, there must be an integer $N=N(\epsilon)$ such that $Z \leq N$ with probability (conditional on $F_{m}$ ) of at least $1-\epsilon / 2$. Let us now replace in (21) the number $\epsilon$ by $\epsilon / 2$, and use the relation $n=n(\omega) \leq Z$. We obtain

$$
\begin{gathered}
1-\frac{\epsilon}{2} \leq \mathbb{P}\left\{\exists n \in\left\{m_{1}, \ldots, Z\right\}, w^{*} \in W^{*} \forall n^{\prime} \geq n: \mathcal{T}\left(n^{\prime}, w^{*}, \epsilon\right) \mid F_{m}\right\} \\
\leq \mathbb{P}\left\{\exists n \in\left\{m_{1}, \ldots, Z\right\}, w^{*} \in W^{*} \forall n^{\prime} \geq n: \mathcal{T}\left(n^{\prime}, w^{*}, \epsilon\right) \wedge Z \leq N \mid F_{m}\right\}+\mathbb{P}\left\{Z>N \mid F_{m}\right\} \\
\leq \mathbb{P}\left\{\exists n \in\left\{m_{1}, \ldots, Z\right\}, w^{*} \in W^{*} \forall n^{\prime} \geq n: \mathcal{T}\left(n^{\prime}, w^{*}, \epsilon\right) \wedge Z \leq N \mid F_{m}\right\}+\frac{\epsilon}{2} .
\end{gathered}
$$

Subtracting $\epsilon / 2$ on both sides yields

$$
\mathbb{P}\left\{\exists n \in\left\{m_{1}, \ldots, Z\right\}, w^{*} \in W^{*} \forall n^{\prime} \geq n: \mathcal{T}\left(n^{\prime}, w^{*}, \epsilon\right) \wedge Z \leq N \mid F_{m}\right\} \geq 1-\epsilon,
$$

and hence

$$
\begin{gathered}
\mathbb{P}\left\{\exists w^{*} \in W^{*} \forall n^{\prime} \geq N: \mathcal{T}\left(n^{\prime}, w^{*}, \epsilon\right) \mid F_{m}\right\} \\
\geq \mathbb{P}\left\{\exists n \in\left\{m_{1}, \ldots, Z\right\}, w^{*} \in W^{*} \forall n^{\prime} \geq n: \mathcal{T}\left(n^{\prime}, w^{*}, \epsilon\right) \wedge Z \leq N \mid F_{m}\right\} \geq 1-\epsilon,
\end{gathered}
$$

which gives the assertion of the Lemma. 
Corollary. With $P_{w^{*}}(n)$ denoting the probability that a fixed agent traverses walk $w^{*}$ in cycle $n$, the following assertion holds: ${ }^{4}$ For each $\epsilon>0$ and $m \in \mathbb{N}$ there is an $N \in \mathbb{N}$ such that

$$
\mathbb{P}\left\{\exists w^{*} \in W^{*} \forall n^{\prime} \geq N: P_{w^{*}}\left(n^{\prime}\right) \geq 1-\epsilon \mid F_{m}\right\} \geq 1-\epsilon .
$$

Proof. We replace $\epsilon$ by $\tilde{\epsilon}$ in Lemma 3.7, and set (with $L$ given by (16))

$$
\tilde{\epsilon}=\min \left(\frac{1}{2 L}, \frac{\gamma \epsilon}{2 d L^{3}}\right)<\epsilon .
$$

By Lemma 3.7, to given $\tilde{\epsilon}>0$ and $m \in \mathbb{N}$, there exists an $N \in \mathbb{N}$ such that with a probability (conditional on $F_{m}$ ) larger or equal to $1-\tilde{\epsilon}$,

$$
\exists w^{*} \in W^{*} \forall n^{\prime} \geq N: \mathcal{T}\left(n^{\prime}, w^{*}, \tilde{\epsilon}\right) .
$$

Assume $\mathcal{T}\left(n^{\prime}, w^{*}, \tilde{\epsilon}\right)$. Then

$$
\tau_{k l}\left(n^{\prime}\right) \leq L\left(w^{*}\right) \tilde{\epsilon} \leq L \tilde{\epsilon} \leq \gamma \epsilon /\left(2 d L^{2}\right) \quad \forall(k, l) \notin w^{*},
$$

and

$$
\tau_{k l}\left(n^{\prime}\right) \geq 1 / L\left(w^{*}\right)-\tilde{\epsilon} \geq 1 /(2 L) \quad \forall(k, l) \in w^{*}
$$

as before. Hence we conclude in an analogous way as in the proof of Lemma 3.6: The probability that a fixed agent proceeds from a node $k \in w^{*}$ to a node $r \notin w^{*}$ is smaller or equal to

$$
d \cdot \frac{\gamma \epsilon /\left(2 d L^{2}\right)}{(1 /(2 L)) \cdot \gamma}=\frac{\epsilon}{L}
$$

As a consequence, the probability that walk $w^{*}$ is traversed by the considered agent is larger or equal to

$$
(1-\epsilon / L)^{L\left(w^{*}\right)} \geq(1-\epsilon / L)^{L} \geq 1-\epsilon .
$$

After these preparations, we are now in the position to prove the main theorem:

Proof of Theorem 3.1. By Lemma 3.1,

$$
\begin{gathered}
\mathbb{P}\left(B_{1} \wedge \ldots \wedge B_{n}\right)=\mathbb{P}\left(B_{1}\right) \cdot \mathbb{P}\left(B_{2} \mid B_{1}\right) \cdot \ldots \cdot \mathbb{P}\left(B_{n} \mid B_{1} \wedge \ldots \wedge B_{n-1}\right) \\
\leq(1-p)^{S}(1-c p)^{S} \ldots\left(1-c^{n-1} p\right)^{S}=\left[\prod_{i=1}^{n}\left(1-c^{i-1} p\right)\right]^{S} .
\end{gathered}
$$

Let us set

$$
w(p, c, S)=\left[\prod_{i=1}^{\infty}\left(1-c^{i-1} p\right)\right]^{S} .
$$

Since $A=\neg\left(B_{1} \wedge B_{2} \wedge \ldots\right)$, we get

$$
\mathbb{P}(A)=1-\lim _{n \rightarrow \infty} \mathbb{P}\left(B_{1} \wedge \ldots \wedge B_{n}\right) \geq 1-\lim _{n \rightarrow \infty}\left[\prod_{i=1}^{n}\left(1-c^{i-1} p\right)\right]^{S}=1-w(p, c, S) .
$$

\footnotetext{
${ }^{4}$ As to the interpretation of $P_{w^{*}}(n)$ as a random variable, cf. Remark 3.1.
} 
Is is easy to see that by choosing $S$ sufficiently large or by choosing $\rho$ sufficiently small, it can be achieved that $w(p, c, S)$ gets arbitrarily small. Hence, by an appropriate choice of $S$ or $\rho$, we can achieve that $\mathbb{P}(A) \geq 1-\epsilon / 4$. Since

$$
\sum_{n=1}^{\infty} \mathbb{P}\left(F_{n}\right)=\mathbb{P}\left(F_{1} \vee F_{2} \vee \ldots\right)=\mathbb{P}(A) \leq 1
$$

there is an integer $\kappa=\kappa(\epsilon)$ such that

$$
\sum_{n=\kappa+1}^{\infty} \mathbb{P}\left(F_{n}\right)<\frac{\epsilon}{4}
$$

SO

$$
\mathbb{P}\left(F_{1} \vee \ldots \vee F_{\kappa}\right)=\sum_{n=1}^{\kappa} \mathbb{P}\left(F_{n}\right)=\mathbb{P}(A)-\sum_{n=\kappa+1}^{\infty} \mathbb{P}\left(F_{n}\right) \geq\left(1-\frac{\epsilon}{4}\right)-\frac{\epsilon}{4}=1-\frac{\epsilon}{2}
$$

Let us replace $\epsilon$ by $\tilde{\epsilon}=\epsilon / 2$ in the Corollary to Lemma 3.7. Then, for each $m \in \mathbb{N}$, there must be an integer $N=N(\epsilon, m)$ such that

$$
\mathbb{P}\left\{\exists w^{*} \in W^{*} \forall n^{\prime} \geq N(\epsilon, m): P_{w^{*}}\left(n^{\prime}\right) \geq 1-\epsilon \mid F_{m}\right\} \geq 1-\frac{\epsilon}{2} .
$$

Let $N(\epsilon)=\max \{N(\epsilon, m) \mid 1 \leq m \leq \kappa\}$. Then also

$$
\mathbb{P}\left\{\exists w^{*} \in W^{*} \forall n^{\prime} \geq N(\epsilon): P_{w^{*}}\left(n^{\prime}\right) \geq 1-\epsilon \mid F_{m}\right\} \geq 1-\frac{\epsilon}{2}
$$

for all $m \leq \kappa$. Consequently,

$$
\begin{gathered}
\mathbb{P}\left\{\exists w^{*} \in W^{*} \forall n \geq N(\epsilon): P_{w^{*}}(n) \geq 1-\epsilon\right\} \\
=\sum_{m=1}^{\kappa} \mathbb{P}\left\{\exists w^{*} \in W^{*} \forall n \geq N(\epsilon): P_{w^{*}}(n) \geq 1-\epsilon \mid F_{m}\right\} \cdot \mathbb{P}\left(F_{m}\right) \\
+\mathbb{P}\left\{\exists w^{*} \in W^{*} \forall n \geq N(\epsilon): P_{w^{*}}(n) \geq 1-\epsilon \mid \neg\left(F_{1} \vee \ldots \vee F_{\kappa}\right)\right\} \cdot \mathbb{P}\left(\neg\left(F_{1} \vee \ldots \vee F_{\kappa}\right)\right) \\
\geq \sum_{m=1}^{\kappa} \mathbb{P}\left\{\exists w^{*} \in W^{*} \forall n \geq N(\epsilon): P_{w^{*}}(n) \geq 1-\epsilon \mid F_{m}\right\} \cdot \mathbb{P}\left(F_{m}\right) \\
\geq\left(1-\frac{\epsilon}{2}\right) \cdot \sum_{m=1}^{\kappa} \mathbb{P}\left(F_{m}\right) \geq\left(1-\frac{\epsilon}{2}\right)^{2} \geq 1-\epsilon .
\end{gathered}
$$

This proves the Theorem.

Corollary. Let $P_{n}$ denote the probability that a fixed agent traverses an optimal walk in cycle $n$. Then for each $\epsilon>0$, it can be achieved by choosing $S$ sufficiently large or by choosing $\rho$ sufficiently small, that for an integer $N(\epsilon)$,

$$
P_{n} \geq 1-\epsilon \text { for all } n \geq N(\epsilon) \text {. }
$$


Proof. Replacing $\epsilon$ by $\tilde{\epsilon}$ and $N(\epsilon)$ by $\tilde{N}(\tilde{\epsilon})$ in Theorem 3.1, we obtain

$$
\forall \tilde{\epsilon}>0 \exists S(\tilde{\epsilon}), \tilde{N}(\tilde{\epsilon}): \mathbb{P}\left\{\exists w^{*} \in W^{*} \forall n \geq \tilde{N}(\tilde{\epsilon}): P_{w^{*}}(n) \geq 1-\tilde{\epsilon}\right\} \geq 1-\tilde{\epsilon}
$$

and an analogous assertion where $S(\tilde{\epsilon})$ is replaced by $\rho(\tilde{\epsilon})$. From $(25)$, the weaker assertion

$$
\forall \tilde{\epsilon}>0 \exists S(\tilde{\epsilon}), \tilde{N}(\tilde{\epsilon}) \forall n \geq \tilde{N}(\tilde{\epsilon}): \mathbb{P}\left\{\exists w^{*} \in W^{*}: P_{w^{*}}(n) \geq 1-\tilde{\epsilon}\right\} \geq 1-\tilde{\epsilon}
$$

follows. (Analogously for $\rho(\tilde{\epsilon})$.) Let us consider agent $A_{s}$. Then

$$
\begin{gathered}
P_{n}=\sum_{j=1}^{R} \mathbb{P}\left(E_{n}^{(s)}\left(w_{j}^{*}\right)\right) \geq \sum_{j=1}^{R} \mathbb{P}\left(E_{n}^{(s)}\left(w_{j}^{*}\right) \wedge P_{w_{j}^{*}}(n) \geq 1-\tilde{\epsilon}\right) \\
=\sum_{j=1}^{R} \mathbb{P}\left(P_{w_{j}^{*}}(n) \geq 1-\tilde{\epsilon}\right) \cdot \mathbb{P}\left(E_{n}^{(s)}\left(w_{j}^{*}\right) \mid P_{w_{j}^{*}}(n) \geq 1-\tilde{\epsilon}\right) \geq(1-\tilde{\epsilon}) \sum_{j=1}^{R} \mathbb{P}\left(P_{w_{j}^{*}}(n) \geq 1-\tilde{\epsilon}\right) \\
\geq(1-\tilde{\epsilon}) \mathbb{P}\left\{\exists w_{j}^{*} \in W^{*}: P_{w_{j}^{*}}(n) \geq 1-\tilde{\epsilon}\right\} .
\end{gathered}
$$

By (26), the last expression is, for $n \geq \tilde{N}(\tilde{\epsilon})$, larger or equal to $(1-\tilde{\epsilon})^{2} \geq 1-2 \tilde{\epsilon}$. Setting $\tilde{\epsilon}=\epsilon / 2$ and $\tilde{N}(\tilde{\epsilon})=N(2 \tilde{\epsilon})=N(\epsilon)$ completes the proof.

Remark 3.4. The Corollary above is distinctly weaker than Theorem 3.1, since it does not make an assertion on the "persistence" of one optimal walk which finally survives. — The special case $R=1$ of the Corollary is the main result (Theorem 4.1) of [13].

\section{Conclusions}

We have shown that the convergence result demonstrated in [13] for the Graph-Based Ant System algorithm also holds on essentially relaxed conditions. Our estimations are coarse and based on worst cases, so they are not suitable at all for deriving assertions on the (average) convergence speed, nor on appropriate parameter values for "number of agents" resp. "evaporation factor". This aspect has been discussed in [13] in more detail. It can be hoped that future investigations will produce results concerning the interesting questions mentioned above. Another open problem is the convergence behavior of Ant-System-type heuristics under completely different trail level update strategies as the one considered here. We conjecture that in the final phase, a strategy resembling "Global-best" must be applied to prevent premature convergence to a suboptimal solution (in a similar way as in Simulated Annealing, the reduction of the temperature parameter is essential for convergence to the optimal solution), but it seems too early to give a definite answer to this question.

Of course, it would be desirable to have convergence with probability exactly one and not only with a probability larger or equal $1-\epsilon$. In [14], almost sure convergence results are shown for suitably modified trail update schemes in the framework of a simplified GBAS variant. It is possible that by using such modified update rules, also the results in the present paper for the "complete" GBAS algorithm might be sharpened to obtain convergence with probability one. 


\section{References}

[1] Aarts, E., Korst, J., Simulated Annealing and Boltzmann Machines, Wiley (1989)

[2] Bullnheimer, B., Hartl, R. F., Strauss, C., "A new rank-based version of the Ant System: A computational study", Central European Journal for Operations Research and Economics 7 (1) (1999), pp. 25-38.

[3] Bullnheimer, B., Kotsis, G., Strauss, C., "Parallelization Strategies for the Ant System", in: R. Leone, A. Murti, P. M. Pardalos, G. Toralo (eds.): High Performance Algorithms and Software in Nonlinear Optimization, Kluwer Academic Publishers, Dordrecht (1998), pp. 87-100.

[4] Dorigo, M., "Optimization, Learning and Natural Algorithms", PhD Thesis, Dept. of Electronics, Politecnico di Milano, Italy (1992).

[5] Dorigo, M., Di Caro, G., Gambardella, L.M., "Ant Algorithms for Discrete Optimization", Artificial Life 5(2) (1999).

[6] Dorigo, M., Di Caro, G., "The Ant Colony Optimization Meta-Heuristic", in: D. Corne, M. Dorigo, F. Glover (eds.), New Ideas in Optimization, McGraw-Hill (1999), pp. 11-32.

[7] Dorigo, M., Maniezzo, V., Colorni, A., "The Ant System: An Autocatalytic Optimization Process", Technical Report 91-016, Dept. of Electronics, Politecnico di Milano, Italy (1991).

[8] Dorigo, M., Maniezzo, V., Colorni, A., "The Ant System: Optimization by a colony of cooperating agents", IEEE Trans. on Systems, Man, and Cybernetics 26 (1996), pp. 1-13.

[9] Feo, Th. A., Resende, M. G. C., "Greedy randomized adaptive search procedures", J. of Global Optimization 6 (1995), pp. 109-133.

[10] Gallager, R. G., Discrete Stochastic Processes, Kluwer (1996).

[11] Gambardella, L.M., Dorigo, M., "Ant-Q: A Reinforcement Learning approach to the traveling salesman problem", Proc. of ML-95, Twelfth Intern. Conf. on Machine Learning (1995), pp. 252260 .

[12] Geman, D., Geman, S., "Stochastic relaxation, Gibbs distributions, and the Bayesian restauration of images", IEEE Proc. Pattern Analysis and Machine Intelligence 6 (1984), pp. 721-741.

[13] Gutjahr, W.J., "A graph-based Ant System and its convergence", Future Generation Computer Systems 16 (8) (2000), pp. 873-882.

[14] Gutjahr, W.J., "ACO algorithms with guaranteed convergence to the optimal solution", Information Processing Letters 82 (2002), pp. 145-153.

[15] Hajek, B., "Cooling schedules for optimal anealing", Mathematics of Operations Research 13 (1988), pp. 311-329.

[16] Holland, J. H., Adaption in Natural and Artificial Systems, University of Michigan Press (1975).

[17] Maniezzo, V., "Exact and approximate nondeterministic tree-search procedures for the Quadratic Assignment Problem", INFORMS J. on Computing 11 (4) (1999), pp. 358-369.

[18] Michalewicz, Z., Genetic Algorithms + Data Structures = Evolution Programs, Springer (1992). 